\title{
LA FILOSOFÍA DE LA BIOLOGÍA DE AUGUSTE COMTE
}

\author{
Marcelo Pérez M. \\ Université Paris I, Sorbonne
}

\section{Resumen}

El propósito de estas páginas es mostrar el estatus epistemológico de la biología en Comte. El lugar que ocupa en la filosofía positiva, poniendo un particular énfasis en su relación con la ciencia sociológica. Desde el dominio de la biología, de su posición enciclopédica y de su filiación con otras ciencias, se desarrollan algunas consideraciones metodológicas de aquel «momento único», donde el estudio de la materia inerte da paso al estudio de los cuerpos organizados.En segundo lugar, se propone mostrar al Comte filósofo de la biología e intentar exponer una idea tanto del conjunto de la filosofía positiva como del positivismo.

Palabras claves: Auguste Comte, filosofía de la biología.

\begin{abstract}
:
Augustecomte's philosophy of biology. The purpose of these pages is to show the epistemological status of biology in Comte: the place of the positive philosophy, paying particular attention to its relationship to the science of sociology. Based on the realm of biology, its encyclopedic position and its affiliation with other sciences, a series of methodological considerations are developed regarding that «unique moment», when the study of inert matter gives way to the study of organized bodies. In the second place I intend to show Comte, the philosopher of biology, and to try to present an idea of positivism as well as of positive philosophy as a whole.
\end{abstract}

Keys words: Auguste Comte, philosophy of biology. 


\section{Introducción}

Veinte años antes de la publicación de Las reglas del método sociológico de Durkheim, en el mes de enero de 1874, el joven chileno Jorge Lagarrigue (18541894) apunta en su diario de vida,

Quiero, poco a poco, tener una noción exacta de la hermosa ciencia de la biología, indispensable preliminar para el estudio de la sociología, o ciencia de las sociedades.

Esta intuición es propia del siglo XIX. Lagarrigue es uno de los comteanos más importantes de Sudamérica. En 1875 realiza la primera traducción al español del Cours de philosophie positive. En el mismo año Lagarrigue entra en dialogo con Emile Littré, lo que precipita su partida a París con la intención de estudiar medicina en la prestigiosa Faculté de médecine, donde alcanzará asistir a las cátedras de Bernard, Robin, Charcot y Vulpian. Lagarrigue se interesa, desde luego, por «la bella ciencia de la biología» y finalmente estudiará «las artes médicas». Sin embargo el Dr. Lagarrigue nunca ofició de médico. Lo interesante es que su iniciación filosófica en Santiago, más sus estudios médicos en la faculté, y sobre todo, su actividad en el seno de la sociétépositiviste resultaron en el apóstol Jorge Lagarrigue, quien durante el periodo de 1883 hasta su fallecimiento en 1894, participó, de la regeneración afectiva, espiritual y moral de las sociedades occidentales, propiciada por una red de iglesias positivistas que articuló esfuerzos provenientes de distintos continentes.

En más de algún sentido, el positivismo es, parafraseando a Emile Littré, « la obra filosófica del siglo XIX », porque supo entregar « a la filosofía el método positivo de las ciencias, y a las ciencias la idea sintética de la filosofía ». Sin ir más lejos, el profundo impacto que tiene el positivismo sobre el pensamiento político, por ejemplo, re-inaugura el sentido histórico del siglo XIX.

En estas páginas, se propone el desarrollo de aquella intuición recogida del diario íntimo de Lagarrigue. Mi propósito es presentar el estatus epistemológico de la biología en Comte, esto es, el lugar que ésta ocupa en la filosofía positiva, poniendo un particular énfasis en su relación con la ciencia siguiente, la sociología. Para esto, haré una breve descripción del dominio de la biología, de su posición enciclopédica, de su filiación con otras ciencias, y desarrollaré algunas consideraciones metodológicas en aquel «momento único», donde el estudio de la materia inerte da paso al estudio de los cuerpos organizados. 
Se pone una especial atención a las cuestiones que tendrán impacto en el nacimiento de la física social, que en 1836 Auguste Comte bautizará como sociología.La idea de este texto es también hacer aparecer al Comte filósofo de la biología e intentar exponer tanto una idea del conjunto de la filosofía positiva como del positivismo.

Los trabajos de Auguste Comte en biología son de los más importantes que haya realizado el filósofo nacido en Montpellier en 1798. De hecho, estos trabajos son imprescindibles para el nacimiento de su sociología. Si bien, la sociología comteana está muy lejos de aquella que funda Durkheim al final del siglo XIX, cabe mencionar que esta última se realiza discutiendo a Comte, pero también, adoptando ciertas cuestiones de la filosofía positiva.

Debo advertir que el positivismo y la biología, en la segunda mitad del siglo XIX, representan un extenso tema que estas páginas no pueden abordar de modo exhaustivo. En el torbellino de movimientos, ideas, escuelas y tradiciones en París; dos grupos de biólogos y médicos del periodo permiten entrar a la distinción, siempre expuesta a enredos pero indispensable, entre la primera y segunda carrera del fundador del positivismo: Augusto Comte. Un primer grupo y principal, lo componen quienes siguen la interpretación de Emile Littré. De esta interpretación, que reconoce sólo al «primer» Comte, dependerá la divulgación del filósofo tanto en Francia como en el resto del mundo. En Chile, J-V Lastarria y Valentín Letelier, los más conocidos adeptos al positivismo, eran, fundamentalmente, herederos de Littré, aunque se reconociesen comteanos y positivistas. Desde París, Emile Littré, Charles Robin y otros, imprimen al campo de las ciencias de la vida una delimitación disciplinar inspirada en la biología comteana. Robin y Littré, en ese orden, serán los principales divulgadores de la filosofía positiva en el terreno de la medicina.

El segundo grupo es el de los positivistas completos, aquellos que construyeron la Religión de la Humanidad. Comte recoge e interpreta la palabra religión como proveniente del latín re-ligare, unir dos veces. Para Comte la religión tiene por función unir individual y colectivamente al hombre (S, II, p. 7). Si comúnmente las religiones son teológicas, la de Comte es sociológica, por ende, Dios está descartado. En el campo de la medicina, los positivistas «completos» se mantuvieron activos tanto en la facultad de medicina de París, en los hospitales y hasta en la medicina legal. Otros, como elDr. Jorge Lagarrigue, se dedicaron a la divulgación del pensamiento religioso de Comte. Sin la preponderancia del grupo «incompleto», los nombres más importantes de los completos en el campo de la biología y la medicina son elDr. Paul Dubuisson; y en el campo teórico y de la propaganda religiosa, el Dr. Audiffrent. 


\section{La biología en la filosofía positiva}

El proyecto fundamental de la filosofía positiva consiste en una revista enciclopédica de las distintas ciencias que se van constituyendo en el tiempo. Estas -según la ley de clasificación de las ciencias de Comte- estudian los fenómenos desde los más abstractos y simples, como los de la astronomía o las matemáticas, hasta los más concretos y complejos, como la biología y la sociología. Para Comte, después de la existencia matemática, apreciada bajo la forma geométrica, se construye una inmensa serie que finaliza en fenómenos cada vez «más particulares, complicados, y más eminentes que los predecesores» (S, III. p. 53), siendo los fenómenos vitales, sociales y morales los del otro extremo de la cadena iniciada por las matemáticas. Esto es que «toda la economía del entendimiento humano se encuentra reglamentada según la ley general del orden real que en todas partes subordina siempre los más nobles fenómenos a los más groseros»; estos últimos, «siempre más simples y regulares». Esta «regla universal establece desde un comienzo la subordinación total del hombre al mundo, como la de cada ser vivo a su medio correspondiente» (S, III. p. 18).

Según la ley de filiación, cada una de las ciencias positivas aportaría a la consagración de la siguiente, aunque cada ciencia tiene su propia autonomía, entiéndase, su propio método. Para Comte los métodos empleados en una ciencia representan la filosofía de esa ciencia. Es famosa ley de los tres estados, cada rama del conocimiento recorrería los tres estados, teológico, metafísico y positivo. Por otra parte, la ley de la modificabidad indica que los fenómenos son cada vez más modificables según su posición enciclopédica: en los fenómenos celestes el movimiento de rotación o el de gravitación tendría nada o muy poca modificabilidad. En cambio, los fenómenos sociales son los más modificables de todos.

Es importante mencionar que para Comte, la filosofía no puede ser reemplazada por la ciencia y el positivismo no tiene, en ningún caso, que ser considerado como un cientificismo. De este modo, filiación,modificabilidady los tres estados (teológico, metafísico y positivo) son leyes teóricas sobre fenómenos intelectuales o especulativos, no son reales. Es también -parafraseando a Comte- «una concepción positiva de nuestra evolución mental», una ley del espíritu humano en la historia. Es en este sentido en que la filosofía positiva no es una ciencia, sino que corresponde más bien a una filosofía hecha para al estudio abstracto del orden exterior, una filosofía de las ciencias.

Resumiendo, las ciencias recorrerían los tres estados al mismo tiempo que su 
desarrollo va de lo simple a lo complejo, y consecuentemente, cada una de estas mismas ciencias aporta al desarrollo de la siguiente. En el caso de la biología, ésta recibe la herencia de las matemáticas hasta la química. Jorge Lagarrigue explica, en su tesis de medicina de 1883, el sistema de la filosofía de Comte en cuanto al lugar de la biología:

que la biología haya devenido en una ciencia positiva; [...] aquello que no pudo suceder antes de que las ciencias que la precedían en la jerarquía científica, es decir, las matemáticas, la astronomía, la física y la química fueran devenidas todas ciencias positivas. Esta evolución preliminar no estuvo terminada hasta el final del siglo pasado [siglo XVIII] con la constitución de la química; es solamente a comienzos de este siglo que los inmortales trabajos del gran Bichat, establecen los fundamentos de la biología positiva. Todos los fenómenos de la vida fueron desde entonces derivados a simples propiedades de los tejidos y de elementos orgánicos.

A partir de la $40^{\circ}$ lección del Cours de philosophie positive, titulada «Consideraciones filosóficas sobre el conjunto de la biología», Auguste Comte se encuentra con uno de los principales desplazamientos «en el orden» $\mathrm{y}$ «en el método» que tiene su primera gran obra filosófica, el Cours. Una vez revisadas «las ciencias cosmológicas» (matemáticas, astronomía, física y química), Comte se da cuenta que en las ciencias de la vida se debe pensar de otra manera. Ya no se trata sólo de razonar un fenómeno «simple», como el calor o la atracción que la tierra ejerce sobre la manzana de Newton, sino que los fenómenos de la vida tienden a parecerse a un evento o a un acontecimiento.

Comte reconoce que «el estudio del hombre y el del mundo exterior constituyen necesariamente el doble y eterno tema de todas nuestras concepciones filosóficas» (C, I, 40 ${ }^{\circ}$, p. 665), y cada cual puede ser utilizado como punto de partida del otro. Esto es simplemente que la actividad cognitiva puede ser realizada del hombre al mundo, o al revés, del mundo al hombre. Tanto la metafísica como la teología, dice Comte, son maneras de conocer que tienen por principio, en cuanto a la explicación de los fenómenos del mundo exterior, el sentimiento inmediato de quien conoce, esto es, un método subjetivo, o dicho de otra manera, el conocimiento del hombre al mundo. En cambio, la filosofía positiva se caracterizaría por «la subordinación necesaria y racional de la concepción del hombre a aquella del mundo»(C, I, 40 , p. 666), esto es el conocimiento del mundo al hombre. No obstante, ambas formas de conocer son complementarias y, una vez llegada «la madurez» en el conocimiento de las ciencias, la forma subjetiva y la objetiva, llegarían a un verdadera conciliación. 
Aunque la gran diferencia entre la forma teológica o metafísica y la positiva, sería el hecho de que en esta última, la forma objetiva es la que prepondera. No obstante, la forma objetiva prepondera sólo en la constitución del conocimiento abstracto; la subjetiva tiene una función normativa y moral, o parafraseando a Comte, la forma objetiva lleva al conocimiento a su verdadera vocación, impidiendo las contemplaciones absolutas e ilimitadas, que reproducen, en el campo de las ciencias, los principales inconvenientes del estado teológico-metafísico (DSEP, p. 76). En el Système, Comte dice que el único vicio del método subjetivo inicial era permanecer en la búsqueda de lo absoluto (S, I, p. 581).

Comte tiene muy buenas expectativas de la biología como una ciencia que aportaría innovaciones importantes a la metodología y de paso, a la filosofía positiva. En 1842, en las lecciones de conclusiones del Cours, Comte considera que la biología protagoniza, más que ninguna otra ciencia, la evolución del espíritu humano estableciendo las bases de un estudio plenamente filosófico del hombre. Esto porque que a partir de la biología, el espíritu sintético prevalece sobre el analítico.

La biología sería la ciencia que desarrolla «espontáneamente la disposición mental más necesaria a las especulaciones sociológicas», esto es, predispone a la síntesis, más adecuada que la «influencia activa y continua de las tendencias dispersivas de la filosofía inorgánica» (C, I, 58 , p. 708). En el primer volumen del Système, Comte dice que «toda síntesis debe ser subjetiva, porque la objetividad permanece analítica», esto es que la objetividad no puede sistematizar, sino que entrega los materiales para que la subjetividad realice una síntesis (S, I, p. 581-2). Al mismo tiempo, para Comte la lógica comparativa, iniciada por la geometría y perfeccionada por teoría de las clasificaciones, representa un rendimiento claro y neto que la biología ofrece a todo el edificio de la filosofía positiva, y en especial, en lo que se refiere al vínculo estrecho ente el método subjetivo y el objetivo. Más aún, la metodología comparativa inspiraría a la metodología histórica de la sociología.

En otro sentido, para Comte, en el conocimiento del hombre y en el del mundo, ha dominado la división entre la filosofía natural y la filosofía moral desde Aristóteles hasta él mismo(S, I, p. 574). Por ejemplo, el espíritu teológico de las religiones monoteístas de Occidente y Oriente, gracias a su «gran poder de organización», supieron conservar sus prerrogativas, privilegiando la filosofía moral por sobre la natural, lo que tuvo por consecuencia la desaceleración del progreso científico como lo muestran los argumentos de San Agustín contra los astrónomos de Alejandría en el caso de la esfera terrestre a partir del caso de las antípodas (C, II, 54º p. 376). 
Cuando el desarrollo de los conocimientos reales, como la astronomía, se enfrenta inevitablemente con el sistema teológico, «el célebre compromiso cartesiano viene a caracterizar una situación evidentemente provisoria quien proclamará el método positivo a toda la filosofía natural», pero con la única reserva de dejar «una vana presidencia teológica-metafísica» a los estudios sociales y morales. Uno de los grandes aportes de Descartes sería para Comte, por una parte, el de quebrar para siempre «la frágil unidad metafísica instituida en el siglo XII»en cuanto a la filosofía natural (C, II, 58 $8^{\circ}$ p. 714), y por otra, el de impulsar la filosofía natural por sobre la filosofía moral, dejando esta última, como el último bastión de los teólogos retrógrados.

La idea de Comte es que la filosofía positiva podrá al fin unir aquella «distinción momentánea» entre filosofía natural y filosofía moral. Es por esto que cuando se encuentra estudiando a «los seres organizados», el razonamiento ya no tiene que ser el mismo que estudiaba la materia o los seres inorgánicos. Si el método científico en las ciencias de la materia demandan un espíritu analítico, en las ciencias de la vida se trata de uno deductivo, esta vez del todo hacia las partes. Esta distinción opera de manera histórica, y a partir de Aristóteles, constatamos la presencia del método comparativo y deductivo para la biología. Para Comte la tipología ha sido «decisiva» en la clasificación zoológica y ha podido servir consecuentemente de modelo taxonómico hasta el presente. En Comte sin embargo, y desde una perspectiva dogmática, la biología debería hacer parte del grupo de la filosofía moral y no de la filosofía natural. Es por esto último que podemos inferir que para Comte los resultados de la biología no son enteramente naturales. Esto porque «naturaleza» en Comte es sinónimo de artificio. La naturaleza es objetiva y subjetiva a la vez. Por lo tanto, la distinción entre natural y moral en el ámbito del conocimiento sería una cuestión transitoria y no definitiva, y de alguna manera, aquella separación termina en la filosofía positiva.

En uno de los cuadros sobre la clasificación de las ciencias, Comte propone una clasificación binaria con dos grandes grupos: (1) cosmología o ciencia del mundo, y (2) sociología o ciencia del hombre. Si la primera contiene a la matemática, la astronomía, la física y la química; la segunda agrupa a la biología, la sociología y la moral. Para Pierre Laffitte, sucesor de Auguste Comte, el segundo grupo llamado «sociología o ciencia del hombre» se compondría entonces de «una parte central, la sociología [propiamente tal] y de una cima, la moral, y de un preámbulo, la biología». La biología, entonces, es el preámbulo tanto de la sociología como de la moral. 
Sin embargo, refiriéndose a los métodos de la biología, y en especial a su posición en la clasificación enciclopédica, Comte dice que la subordinación de ésta a las ciencias del mundo exterior, esto es, a las ciencias cosmológicas, constituye «el primer fundamento necesario para la positividad racional» $\left(\mathrm{C}, \mathrm{I}, 40^{\circ}, \mathrm{p} .667\right)$. $\mathrm{La}$ idea de una subordinación de la biología a una analítica propia de la cosmología, para Comte, es sinónimo de una etapa preliminar y fundamental del estudio de las leyes vitales, aunque, finalmente, la biología tendrá su propio método distinto al de las demás. En un uso particular del término «materialismo», para Comte significa reduccionismo, y este se emplea cada vez que quiere denunciar el reduccionismo de una ciencia sobre otra. En un ejemplo, para su época, Comte denuncia la «usurpación» de las matemáticas sociales por parte de Condorcet y Quetelet, quienes reducirían la complejidad social a un modelo metodológico que tiene su especificidad sólo «en la más antiguaciana y perfecta de las ciencias», las matemáticas -para Comte- esta ciencia tiene por fin la medida abstracta del espacio $\left(\mathrm{C}, \mathrm{I}, 3^{\circ}, \mathrm{p} .66\right.$. $)$ y no el estudio de las sociedades. Si de manera general las matemáticas tienen por método el conocer la dimensión de aquello que sólo tiene medida; las sociedades, reducidas a esa forma de medida, ante todo abstracta, tendrían como resultado una idea inapropiada de sí mismas. De la misma manera Pierre Laffitte denuncia el materialismo de la biología. Este consistiría en subordinar la sociología a la biología, donde la primera sería una simple deducción de la ciencia de los seres vivos.

Todas estas precauciones no inhabilitan el hecho que Comte piense en la unidad de todas las especulaciones positivas. De hecho, ese es el nudo central de su filosofía. Por sus ubicaciones en el esquema enciclopédico, las matemáticas son la base de la racionalidad positiva, aunque su influencia o la supremacía de las matemáticas produce una precaria y estéril unidad, «fundada sobre quimeras y vagas hipótesis». Por el contrario, en el otro extremo, la sociología representa el punto de vista humano, «el estado verdaderamente positivo». Así la gran innovación en la construcción enciclopédica de la segunda carrera de Comte, es el momento en que un nuevo método, el subjetivo, otorga la presidencia de las ciencias a la sociología que, posteriormente, reglamenta a todas las demás. En 1848 Comte dice que la ciencia social no sólo es la más importante de todas sino que ésta entrega el único vínculo, lógico y científico, que comportaría el conjunto de nuestras contemplaciones reales (DSEP, p. 43-44). De este modo, comenzando por la teoría cerebral, la sociología fija los fines y el objeto del conocimiento de toda la cadena de las ciencias. De esto modo así, por ejemplo, la sociología no considera el conocimiento de las galaxias que se encuentran a millones de años luz por cuanto este saber no aportaría en nada al problema humano. 
«El conocimiento del universo debe ser estudiado, no por sí mismo, sino por el hombre, antes bien, por la humanidad» (DSEP, p. 76).

\section{La biología en Comte}

El estudio de la biología es especialmente fecundo para las aspiraciones de unidad objetiva y subjetiva del positivismo. La biología de Comte entra en estrecha relación con la sociología, y ésta es, en Comte, mucho más que la ciencia del hecho social.

Para Comte, la biología estudia la vida en su particularidad. Esto quiere decir que los cuerpos individuales logran concebirse como un objeto independiente a la luz de sus propias leyes generales. En el Cours de philosophie positive, el término de biologie, es utilizado por Comte para designar la ciencia abstracta de un objeto general: las leyes vitales; a la vez de una ciencia sintética de una actividad fundamental: la vida. Para Canguilhem, «la invención del término biología fue la expresión de la toma de conciencia, de médicos y fisiólogos de la especifidad de un objeto de investigación que escapa a toda analogía esencial con el objeto de las ciencias de la materia». La solidaridad de la biología comteana para con la sociología es contraria a la de Herbert Spencer o Rene Worms. Para el grupo «organicista» de la revuesociologiqueinternationale(Worms, Le Bon, Le Play, Tarde, Novicow, Kovalewsky, etc.), tanto como para el darwinismo social, el modelo de los seres vivos indica la metodología a seguir en la investigación social.Comte supone que es la sociología quién sanciona el objeto de la biología, aunque le deba a ésta la abstracción de su unidad fundamental: el hombre. Esto, porque para Comte el hombre no existe, es sólo la humanidad quien tiene una existencia real. El hombre que estudia la biología es ante todo una abstracción (DSEP).

Los principales comentaristas del pensamiento de Comte están de acuerdo en que éste es particularmente rico en biología e influyente en medicina. El maestro de Comte en biología es Henri Marie Ducroay de Blainville (1777-1850), «el más digno sucesor de Lamarck» (S, I, p. 571.) de quien hereda la idea que la vida es un fenómeno general interno y continuo de composición y descomposición que se realiza en un organismo determinado, colocado en un medio conveniente (C, I, $40^{\circ}$ p. 680 ).

La definición de la vida como «la correlación entre un organismo apropiado en un medio conveniente», nace de la controversia que Comte plantea a Xavier Bichat (1771-1802). Para este último la vida es «el conjunto de funciones que se resisten a la muerte». Para Comte, Bichat es el primero en establecer la biología sobre una 
base positiva. Lo instala como un momento bisagra entre la especulación metafísica y la ciencia verdaderamente positiva. Sin embargo, para el autor del Cours, Bichat habría cometido el error capital de continuar una preocupación propia de la antigua filosofía, cual es, «la falsa idea de un antagonismo absoluto entre naturaleza muerta y la naturaleza viviente». Bichat habría elegido, «aquella quimérica lucha por el carácter esencial de la vida». Esta «profunda irracionalidad» consiste en suprimir uno de los dos elementos inseparables de la armonía subyacente a la vida. Esta armonía es la que se da entre el ser viviente y el medio; y por ende, esta relación, es la condición fundamental de la vida $\left(\mathrm{C}, \mathrm{I}, 40^{\circ}\right.$ p. 665$)$.

Por otra parte, las dos grandes ramas de la biología, la anatomía y la fisiología, tratan del órgano y de la función. Entonces, a la correlación de un medio con su organismo, es necesario agregarle una idea de función (Cours II, $60^{\circ}$, p. 682). La función, como es trivial en los años de Comte, representa el acto de una estructura. Dicho de una manera especial para la biología, es el correlato dinámico de una estructura anatómica. Para Comte es preferible asignar una estructura a una función, que dejar una estructura en la indeterminación.De manera general, una función en biología es alentada y estimulada por un «simple» racionamiento matemático. Comte dice que «siendo posible el conocimiento a priori del medio ambiente», esto es «a partir del conjunto de las otras ciencias fundamentales» [las cosmológicas], un problema biológico puede ser evocado, «siguiendo un enunciado, el más matemático posible, en sus términos más generales: dado un órgano o una modificación orgánica, encontrar la función o el acto y recíprocamente» (Cours I, L 40 683-684). El énfasis de la cuestión de la estructura y de la función va a dominar el desarrollo en biología como en sociología hasta llegar tanto a la moral como a la política. Según Georges Canguilhem, Comte concibe el movimiento como subordinado al equilibrio, la dinámica como lógicamente fundada por la estática, cuestión desarrollada primero por D'Alambert y luego por Lagrange.

Si como vimos Comte quiere poner fin a la distinción entre filosofía natural y filosofía moral. Para el filósofo autor del Système, la historia humana, desde el punto de vista físico, intelectual y moral, está convocada a terminar las disposiciones naturales del hombre pero no a constituirlas. En otro sentido, para el positivismo religioso de Jorge Lagarrigue la ley de los tres estados fija un modelo y un principio de las leyes del progreso teniendo en cuenta el a priori que la biología le impone a la historia. Este a priori biológico coincide con un tiempo en el cual se desarrolla la positividad de la biología. Jorge Lagarrigue, corroborando su intuición de 1874, explica en su tesis que si en la antigüedad el desarrollo del espíritu positivo fue principalmente 
producto de las nociones matemáticas y astronómicas; en la edad media estos emanaron preferentemente de las especulaciones físicas y químicas. En los tiempos propiamente modernos, la última preparación debía depender esencialmente de los estudios en biología. De hecho el positivismo de la segunda carrera de Comte, el religioso, comienza con el estudio del hombre abstracto a partir de la biología, particularmente a través de la fisiología cerebral.

\section{La fisiología cerebral.}

La $45^{\circ}$ lección del Cours tiene por objeto la teoría positiva de las funciones intelectuales y morales. Es allí cuando Comte, influenciado por las clasificaciones de Spurzheim y Gall muestra la existencia de facultades (funciones), afectivas e intelectuales, y su pista físico-orgánica, su estructura, estaría localizada en el cerebro.Según Comte después de F-J Gall (1758-1828) habrían tres verdades que se habrían establecido: $1^{\circ}$ que el cerebro es la sede de todas nuestras facultades mentales, $2^{\circ}$ que el cerebro no es un órgano, sino un aparato; pero un aparato (un conjunto de órganos) predispuesto a funciones especiales, y $3^{\circ}$ que las facultades cerebrales son innatas y plurales.

Comte desarrolla desde 1846 las funciones fisiológicas del cerebro humano a modo de un cuadro sistemático sobre el alma humana. A partir de Gall, Comte sanciona que el cerebro mantiene funciones afectivas, mentales y motoras. Estas tres serían el «alma positiva». Comte como Gall, inscriben su investigación en una tradición que va de la filosofía moral hasta la frenología. Para Comte aquella tradición va de Aristóteles (el tratado Del alma) a San Pablo (la Naturaleza y la Gracia), como de Hobbes a los enciclopédistas: Diderot, D'Alembert, D'Holbach; pasando por A treatise of human nature (1740) del filósofo escoses David Hume, y sobre todo por Le rapport du physique et du moral de l'homme (1802) de Cabanis que sería la gran obra antes de Anatomie et physiologie du systèmenerveux en général et du cerveau en particulier (1810) de Gall, que según Canguilhem, es el inicio de la ciencia del cerebro.

El lugar anatómico del alma es entonces el aparato cerebral. Es en este sentido, y siguiendo a Laurent Clauzade, que debe leerse la fisiología cerebral comteana, es decir el estudio de las funciones sentimentales, intelectuales y prácticas la principal aplicación de la frenología cerebral es sociológica: ella entrega a la estática social una suerte de modelo de funcionamiento cerebral que permite una formalización biológica de cuestiones sociológicas. Esta formalización biológica le permite a Comte 
sostener una idea estática del hombre que puede ser desarrollada como una idea de orden social. Si en biología Comte subordina la estructura a la función, en sociología será en un sentido inverso, el progreso es el desarrollo del orden. Esto permite que aquella idea del hombre abstracto contenga su propio modelo de desarrollo.

En el Cours, y en su primera carrera, Comte sistematiza el conjunto de las ciencias fundamentales, en la construcción religiosa del Système, sistematiza la existencia humana en su triple dimensión: afectiva, intelectual y práctica. Desde 1851 Comte agrega una séptima ciencia, la moral. Con la moral, las ciencias se vuelven una verdadera doctrina: «pudiendo reglamentar nuestros sentimientos, nuestros pensamientos y nuestros actos».

Del encuentro con Clotilde de Vaux, la musa inspiradora de la religión positivista, Comte extrae la idea de que la inteligencia no debe reinar sino servir. En el discurso de 1848 el espíritu (la inteligencia) debe ser el primer ministro del corazón y jamás su esclavo (DSEP, p. 60). Comte declara la preponderancia del sentimiento por sobre la inteligencia y la acción. No obstante, como el corazón es ciego, primero es necesario sistematizar los pensamientos y sólo después los sentimientos y la acción. Para Comte el exterior es el modelo para ordenar el interior. Esto es que el espíritu busca en el conocimiento de los fenómenos que le son exteriores, la manera de reglamentarse a sí mismo. No obstante, si la preponderancia de lo humano es el sentimiento, entonces el método debe ser necesariamente el subjetivo. Comte desarrolla un cuadro con 18 funciones afectivas, intelectuales y motoras que sintetizan su fisiología cerebral (S,I, p.XX). En cuanto a las funciones afectivas, ellas se dividen entre egoístas y altruistas. Estas últimas son esenciales para su definición de Humanidad como «el conjunto de los seres convergentes, pasados, presentes y futuros»(Cat, p. 67). Para Comte, el altruismo de vivir el gran día, de vivir para los demás son el resultado de las condiciones de existencia propias de la «naturaleza humana», esta última convenida después de la abstracción realizada sobre la cadena total de los seres organizados, es decir, tras la biología.

Es en este sentido que la biología se establece y estabiliza, luego de que la sociología y la moral le indiquen su objeto y destinación. Tal como habíamos mencionado, la vida es esa doble relación de un ser con un medio, para de Blainville, un proceso de asimilación y desasimilación. Lo propio de la teoría cerebral comteana son las condiciones anatómicas y fisiológicas necesarias al cumplimiento de funciones sociales (además, desde el punto de vista lógico y científico, para Comte, la noción de anatomía presupone la noción de función, sino ella flota indeterminada). 
Este punto nos conduce al inicio de la carrera del joven Comte, quien en 1822 concuerda también en coincidir el hombre biológico con el histórico:

«La física social, es decir, el estudio deldesarrollo colectivo de la especie humana es realmente un brazo de la fisiología, es decir del estudio del hombre, concebido en toda su extensión. En otros términos, la historia de la civilización no es otra cosa que el seguimiento y el complemento indispensable de la historia natural del hombre» (Plan, p. 155).

\section{Bibliografía}

BOURDEAU, Michel, «La posteridad sociológica de Auguste Comte: Lo normal y lo patológico en Durkheim». EMPIRIA. Revista de Metodología de Ciencias Sociales. Madrid: $\mathrm{N}^{\circ} 16$, julio-diciembre, 2008.

BRAUNSTEIN, Jean François. La philosophie de la médicined'Auguste Comte. Paris: PUF. 2009.

CANGUILHEM, Georges, PIQUEMAL, Jacques, ULMANN, Jacques. (1962) Du développement à l'évolution au XIXe siècle. Paris: PUF. 2003.

CANGUILHEM, Georges (1968). Etudes d'histoireet de philosophies des sciences.Librairie J. Paris: Vrin. 1989.

CLAUZADE, Laurance. L'organe de la pensée. Biologieetphilosophie chez. Auguste Comte. Besançon : Presses Universitaires de Franche-Comté. 2009.

CLAUZADE, Laurance. «La notion de fonctiondans la philosophiebiologiquecomtienne». En: Revue philosophique de France etl'étranger. Paris:PUF, N4, 2007.

COMTE, Auguste (1830-1842). Cours de philosophie positive.Paris:Hermann 2 volumes. Paris. 1975.

COMTE, Auguste (1852). CatéchismePositiviste. Paris:E. du Sandre. 2009.

COMTE, Auguste (1851-1854) Système de politique positive. 4 Vol. Paris: SociétéPositiviste. 1929.

COMTE, Auguste (1848) Discourssurl'ensemble du positivisme, Paris : Flammarion, 1998.

COMTE, Auguste (1822). Plan de travauxs cientifique nécessaires pourré organiser la société. Paris: L'Harmattan. 2001. 
DE BLAINVILLE, Henri-Marie. De l'Organisation des animaux, ouPrincipesd'anatomiecomparée. Paris:Levrault. 1822.

DHOMBES, Jean. «L'analogieDans les matematiquesselon Comte». En: Revuephilosophique de la France et de l'étranger. Philosophie des sciences: Auguste Comte. Paris: №4. PUF. 2007.

DUBUISSON, Paul. La theoriecerebrale, safondation, son developpement, ses applications. Paris:V. Goupy. 1880.

FEDI, Fernand. «Le prince des philosophes :Aristotevu par Auguste Comte». En Aristoteau XIX siècle. Lille: PressesUniversitaires du Septentrion, 2004.

LAFFITTE, Pierre. «Plan de Cours de biologie». En: RevueOccidentale, Paris: Vol. XI. 1883.

LAGARRIGUE, Jorge. Contribution a l'étude de l'influence du moral sur le physiqueouInfluence du systèmenerveux sur la nutrition. Paris: Thèsepour le doctorat en Médecine. Faculté de Médecine de Paris. 1883.

LAGARRIGUE, Jorge. «Diario íntimo». Inédito. 1875. Santiago: Fondo de la Fundación J-E Lagarrigue.

LAGARRIGUE, Juan Enrique (1884), La Religión de la Humanidad. Santiago:Fundación J-E Lagarrigue. 1947.

LITTRÉ, Emile. Auguste Comte et la philosophiepositiviste. Paris: Hachette. 1863.

PICKERING, Mary. Auguste Comte. An Intellectual Biography.Vol. 3. Cambridge: Cambridge University Press. 2009.

REVUE DE PHILOSOPHIE POSITIVE. Paris: Tome XVI. 1876.

REVUE DES SCIENCES PHILOSOPHIQUES ET THÉOLOGIQUES, Auguste Comte et la religion positiviste, Paris: Vrin, 2003. 